\title{
Spatiotemporal dynamics in a transition zone: patchiness, scale, and an emergent property
}

\author{
M. Anand ${ }^{1}$ and B. L. $\mathrm{Li}^{2}$ \\ ${ }^{1}$ Department of Biology, Laurentian University, Sudbury,Canada. Phone: 1705-675-1151 x2213, \\ Email:manand@nickel.laurentian.ca \\ ${ }^{2}$ Department of Botany \& Plant Sciences, University of California at Riverside, USA. Phone: 1 909-787-4776, \\ Email: bailian@faculty.ucr.edu
}

Keywords: Autocorrelation, Bouteloua eripoda, Bouteloua gracilis, Ecotone, Grassland, Hurst exponent, Population, Variance, Vegetation.

\begin{abstract}
Ecological transition zones are believed to be unique in their ability to shed light on the organization of populations and communities. In this paper, we study vegetation dynamics in the Great Plains short-grass steppe and Chihuahuan desert grassland ecotone in New Mexico, USA, using long-term, high resolution transect studies of the Sevilleta Long-Term Ecological Research Program. We focus on spatial pattern and examine this in several ways: patch size distribution, spatial autocorrelation analysis, and fractal scaling. These methods are used to examine patch size distributions in two sites representing distributional limits of the dominant species and for detection of an emergent scaling property. We found no characteristic spatial resolution (quadrat size), but rather a fractal structure of spatial variation in abundance and a trend towards consistency of the pattern in time when species were closer to their distributional limit. In this, we were able to detect a robust power law behaviour (the emergent property), indicating strong spatial organization via anti-persistence. Our investigation was exploratory in nature; we feel the results are highly suggestive of intrinsic organization in ecological dynamics and may also be useful in generating testable hypotheses regarding the behaviour of species along ecotones.
\end{abstract}

\section{Introduction}

An ecological transition zone (or ecotone) has been defined as a 'zone of transition between adjacent ecological systems, having a set of characteristics uniquely defined by space and time scales and by the strength of the interactions between adjacent ecological systems' (di Castri et al. 1988). In their simplest form, transitions arise from a direct response of ecological systems to discontinuous environmental gradients. In more complicated scenarios (e.g., Loehle et al. 1996, Li 2001a,b), transitions can reflect intrinsic and highly organized dynamics of component response variables and emergent properties (properties which cannot be predicted from knowledge of lower-level properties). Many authors have pointed out that characterization of transition zones can be a very subtle science and most agree that pattern analysis at various hierarchical levels and spatial scales is recommended (Fortin 1994, Gosz and Sharpe 1989, Gosz 1993, Li 2000, 2001a).
Existing hypotheses regarding vegetation dynamics in transition zones focus on spatial patterns of patch sizes. It has been suggested, for example, that sizes of homogenous patches for species in transition zones decreases (with respect to adjacent biomes), and that the variety of patches increases (Delcourt et al. 1983, Gosz 1993). However, other aspects of dynamics, such as spatial autocorrelation, temporal stability, and scaling relations may help to characterize the zones and provide further insight into making the distinction between specific and local patterns and generalizable emergent properties.

In this paper, we study the ecotone occurring between the Great Plains Short-Grass Steppe and the Chihuahuan Desert in New Mexico, USA, which has been monitored for over 10 years as part of the Sevilleta Long-Term Ecological Research program. This ecotone is fairly simple in terms of species richness alone: two grass species, Bouteloua eripoda (a typical Chihuahuan desert species) and Bouteloua gracilis (a typically Great Plains species), intermingle to dominate the vegetation landscape. We pose simple questions about the ecotonal dynamics: 1 . 
How do spatial dynamics of the dominant species change as they move away from their distributional limits? 2. Are these spatial dynamics stable in time?

We focus on the consistency of spatial pattern in time. This requires elaboration since spatial pattern has several components and can be assessed in different ways. The two components of spatial pattern of interest were (i) patch size distribution and (ii) distribution of patches in space (distance between patches). Because of the fine spatial resolution of sampling, both these can be determined directly from this particular dataset. However, we were also interested in applying higher-order analytical methods of spatial pattern detection in vegetation data to compare observed patterns (patch distributions) with those detected by the analytical methods (spatial autocorrelation, quadrat variance and Hurst scaling).

\section{Site and data description}

The Sevilleta Long-Term Ecological Research (LTER) site is located in central New Mexico, USA. Annual mean precipitation is $280 \mathrm{~mm}$, with considerable inter-annual variability. Summer precipitation occurs in intense, local thunderstorms and accounts for over half of the annual moisture. Winter precipitation is unpredictable. Mean monthly temperatures range from $2.5^{\circ} \mathrm{C}$ to $27^{\circ} \mathrm{C}$ (Gosz 1991). The region is a transition zone between the biomes of the cold desert of the Great Basin, the warm Chihuahuan desert, and the semiarid short-grass steppe (Gosz 1991). The two sites chosen for this study were Deep Well and Five Points. They represent two points along the gradient from Chihuahuan desert to Great plains short-grass steppe, with Deep Well occurring north

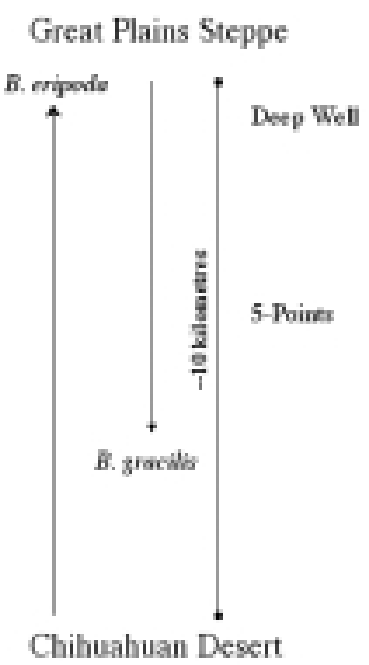

Figure 1. Distribution limits of B. eripoda and B. gracilis in the Sevilleta LTER site (after Gosz 1993) and relative location of sites Deep Well and Five Points. of Five Points (Figure 1). Two perennial C4 grasses, the caespitose blue grama (Bouteloua gracilis) characteristic of the short-grass steppe and the stoloniferous black grama (Bouteloua eripoda) characteristic of the Chihuahuan desert, are co-dominant in both sites, with $B$. eripoda generally occurring with higher cover.

The data we used come from line-intercept studies running from 1989 until 1998. Details about sampling design can be found at http://sevilleta.unm.edu/research/ local/plant/transect/. The line-intercept transects in this study were established to evaluate temporal and spatial dynamics in vegetational transition zones (e.g., black grama grassland/creosote shrubland) at a 1 centimeter resolution. The transects were sampled twice, once in late May or early June and once in late July or early August to monitor potential responses in "cool season" and "warm season" plants. We chose the spring counts, since they were done in the most consistent fashion. Records of plant frequency for the two dominant species $B$. gracilis and B.eripoda, within $10 \mathrm{~cm}$ cells along a $400 \mathrm{~m}$ transect (Transect 1) were used.

\section{Definition of spatial pattern}

We considered three aspects of spatial dynamics. The first was observed patch size distribution. A patch was defined as a contiguous series of occurrences (presence/absence) of a species along the transect. Patch size distributions were fit to well-known theoretical distributions (e.g., normal, exponential, lognormal). Furthermore, changes in patch size distribution were assessed using mean and standard deviation of patch sizes and randomization testing (de Patta Pillar 2001). The second aspect of spatial dynamics considered was spatial autocorrelation (Upton and Fingleton 1985) at various lags (intervals of space between related events). The third was resolution of sampling unit (quadrat variance). Here, the sampling unit was increased by pooling of contiguous quadrats along the transect. Of interest in this case was how variance in species abundance (frequency) between quadrats changed with increasing quadrat size. This has been traditionally used in plant ecology for the detection of critical scales and/or patch size in vegetation (cf. Greig-Smith 1979, 1983). All calculations and analyses were done for all of the 10 years.

\section{Results}

Typical spatiotemporal dynamics of $B$. eripoda and $B$. gracilis are shown in Figure 2. Spatial patterns are not visible from the raw data. The distribution of patch sizes of $B$. eripoda and B. gracilis in both sites for all years is shown in Figure 3. All but five distributions were best fit 

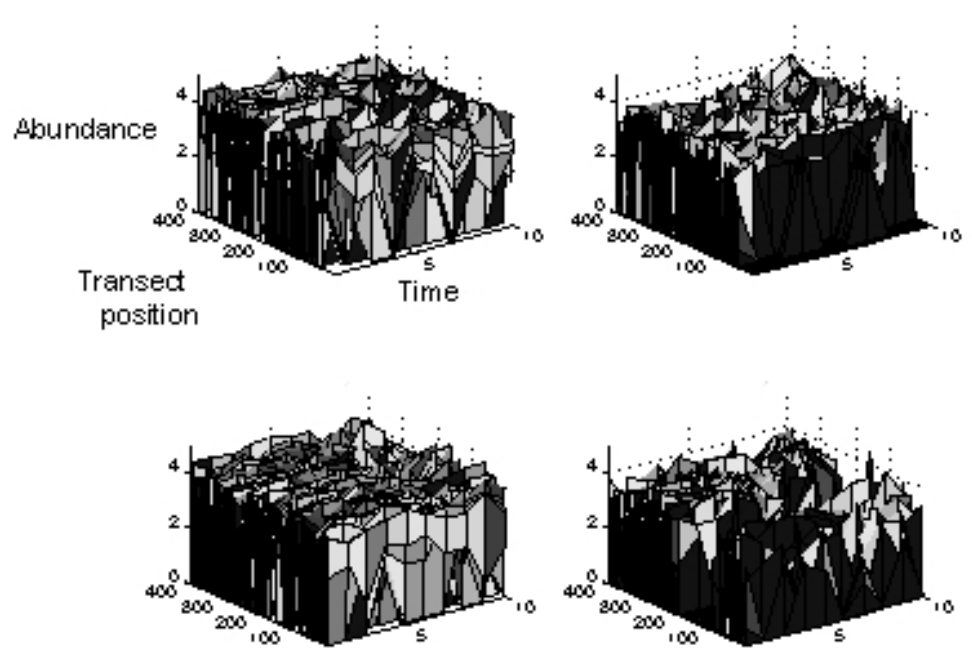

B. eripoda

\section{B. gracilis}

Figure 2. An attempt to visualize spatiotemporal dynamics of B. eripoda and B. gracilis. Abundance (frequency) is estimated within $100 \mathrm{~cm}$ intervals along the transect (coarser scale than analyses). Intensity of shading reflects abundance (dark, high; light, low). Time is in years. Upper graphs are for Deep Well; Lower graphs are for Five Points. The graph shows the difficulty in detecting spatiotemporal patterns visually. by the lognormal distribution (Kolmogorov's $D$ test; $\mathrm{p}<$ .01 ) with similar parameters for $\mu$ and $\sigma$. The exceptions were found in Deep Well, but only one of these did not significantly prove to display a lognormal distribution: $B$. eripoda in $1990(\mathrm{p}<.03), 1995(\mathrm{p}<.11)$ and $1996(\mathrm{p}<$ $.03)$, B. gracilis in $1990(\mathrm{p}<.04)$ and $1992(\mathrm{p}<.03)$.

If we consider the mean and standard deviation of patch size, distributions of both species in both sites changes with time (Figure 4). In Deep Well, significant changes $(\mathrm{p}<.01$, from randomization testing) in mean patch size for B. gracilis occurred in Year 2 (1990), Year 6 (1994), Year 7 (1995) and Year 10 (1998). Significant changes $(\mathrm{p}<.01$, from randomization testing) in mean patch size for B. eripoda occurred in Year 2 (1990), Year 4 (1992), Year 5 (1993), Year 7 (1995) and Year 10 (1998). In addition, it is worth noting that in Deep Well, standard deviation of patch size was slightly lower than its mean for $B$. gracilis, but much higher than its mean for $B$. eripoda. This is interesting because in Deep Well $B$. eripoda is approaching its distributional limit.

In Five Points, significant changes $(\mathrm{p}<.01$, from randomization testing) in mean patch size for B. gracilis occurred in Year 6 (1994), Year 8 (1996), Year 10 (1998). Significant changes $(\mathrm{p}<.01$, from randomization testing) in mean patch size for B. eripoda occurred in Year 2 (1990), Year 4 (1992), Year 9 (1997) and Year 10 (1998). In addition, it is worth noting that in Five Points, standard deviation of patch size in both species was quite different from that in Deep Well. B. gracilis began with standard deviation more or less equal to the mean, but in later years, this increased dramatically. In B. eripoda, standard deviation remained more or less close to its mean. In Five Points, B. gracilis is approaching its distributional limit.

A typical distribution of inter-patch size (distance between patches/areas where either one or both dominant species is absent) is shown in Figure 5 along with its fit to a lognormal distribution $(\mathrm{p}<.01)$. What should be noted here is the fact that the distribution of inter-patches follows roughly the same shape as that of patches, but is not necessarily the same as the patch size distribution of species. In this case (Five Points in 1989), mean inter-patch size of B. eripoda was 22.3 (s.d. 19.8), which was similar to its patch size distribution (Figure 3). However, mean inter-patch size of that of B. gracilis was 17.1 (s.d. 18.7), which was very different from its patch size distribution (Figure 3).

The effect of changing block size on variance (noncentred second moment) is shown in Figure 6. Log-log plots were made because a simple power law relationship was observed between variance and block size. Similar to the calculation of a scaling relation using semivariance (Berry and Lewis 1980, Burrough 1981), this scaling relation could be estimated as the following power law:

$\sigma^{2} \propto l^{2 H}$

In (1), $H$ is the slope of the log-log graph and the Hurst exponent (Hurst 1951, Peitgen et al. 1992). In a purely random structure, whose variables are not correlated, $H$ is found to be 0.5 . When $H$ is greater than 0.5 , the structure shows persistence, namely, the presence of long-range 


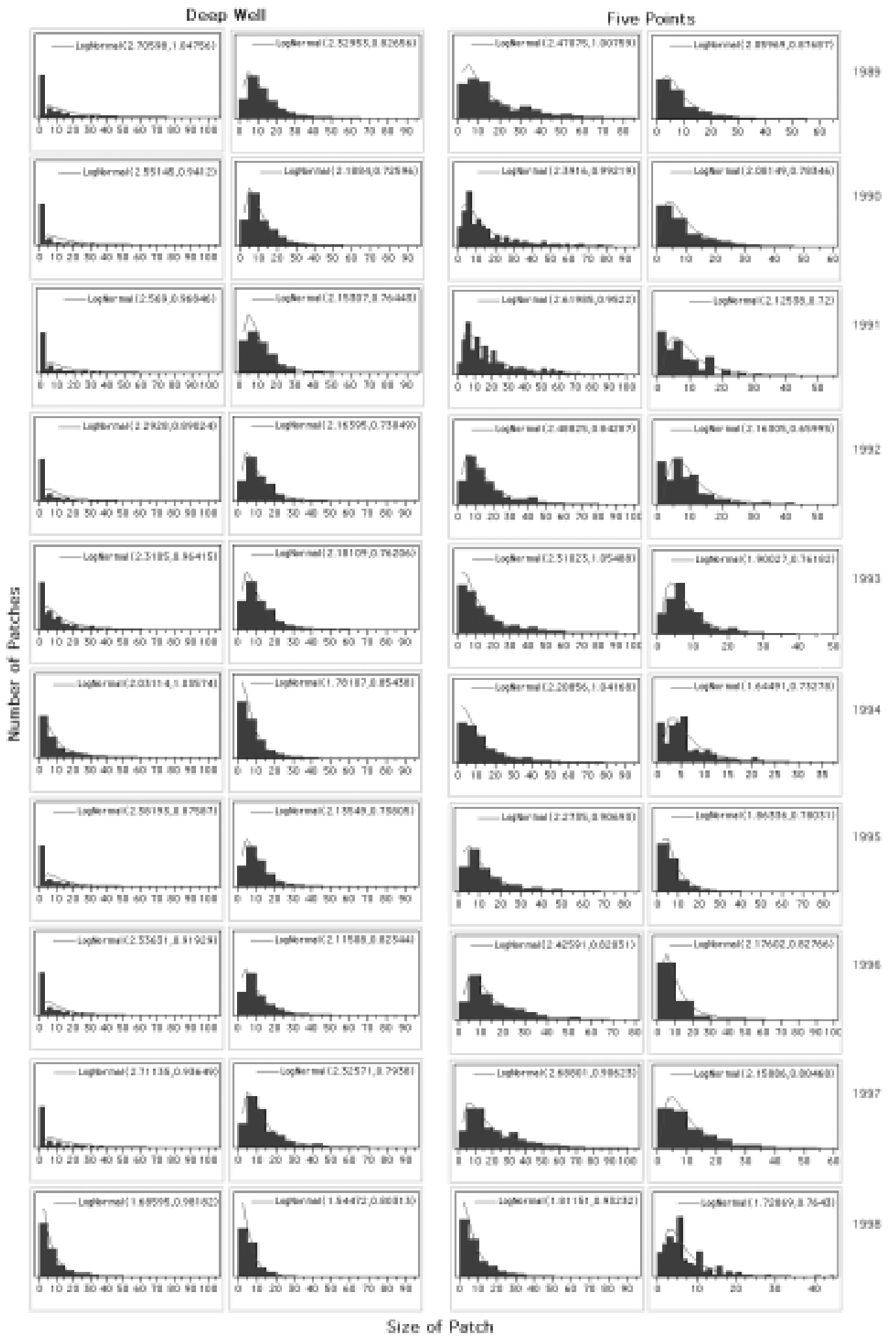

Figure 3. Patch size distribution of B. eripoda (left column) and B. gracilis (right column) from 1989 to 1998 in Deep Well and Five Points. Patch size is based on $10 \mathrm{~cm}$ width classes: 1 corresponds to $0-10 \mathrm{~cm}$ width, 100 corresponds to $990-1000$ $\mathrm{cm}$ width. Fitted curves are lognormal with parameters given $(\mu, \sigma)$. All but one distribution provided statistically good fits. 


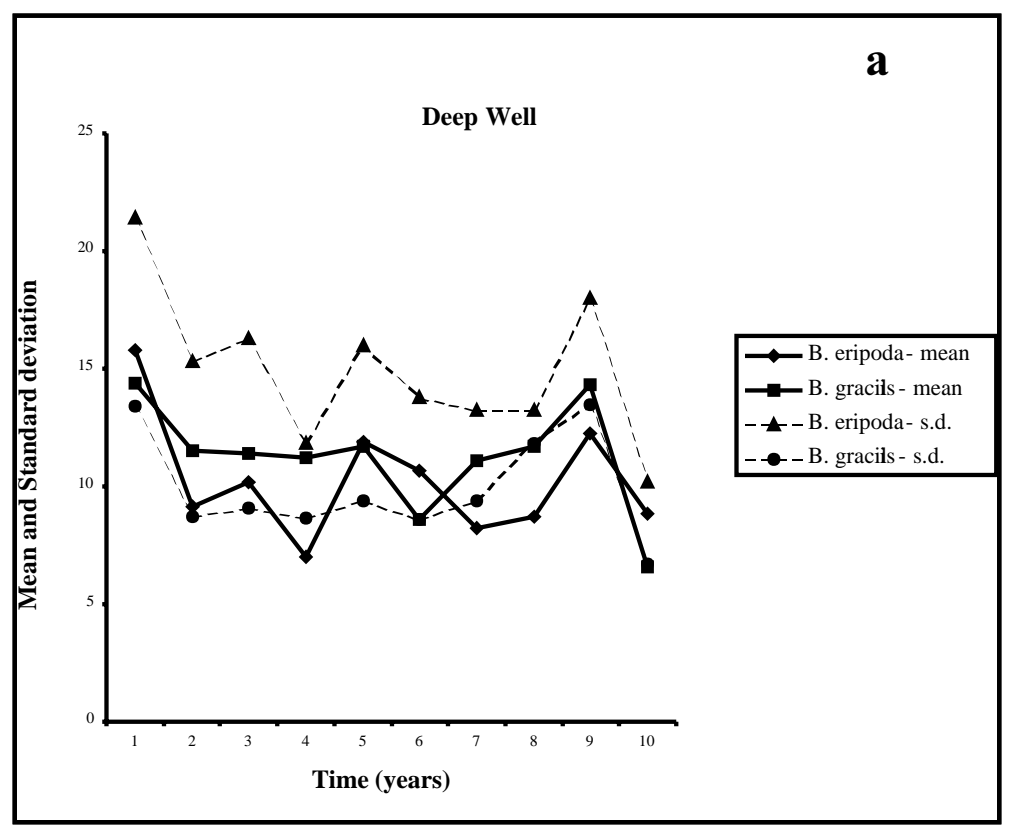

Figure 4. Mean and standard deviation of patch size $(\mathrm{cm})$ for both species in (a) Deep Well and (b) Five Points.

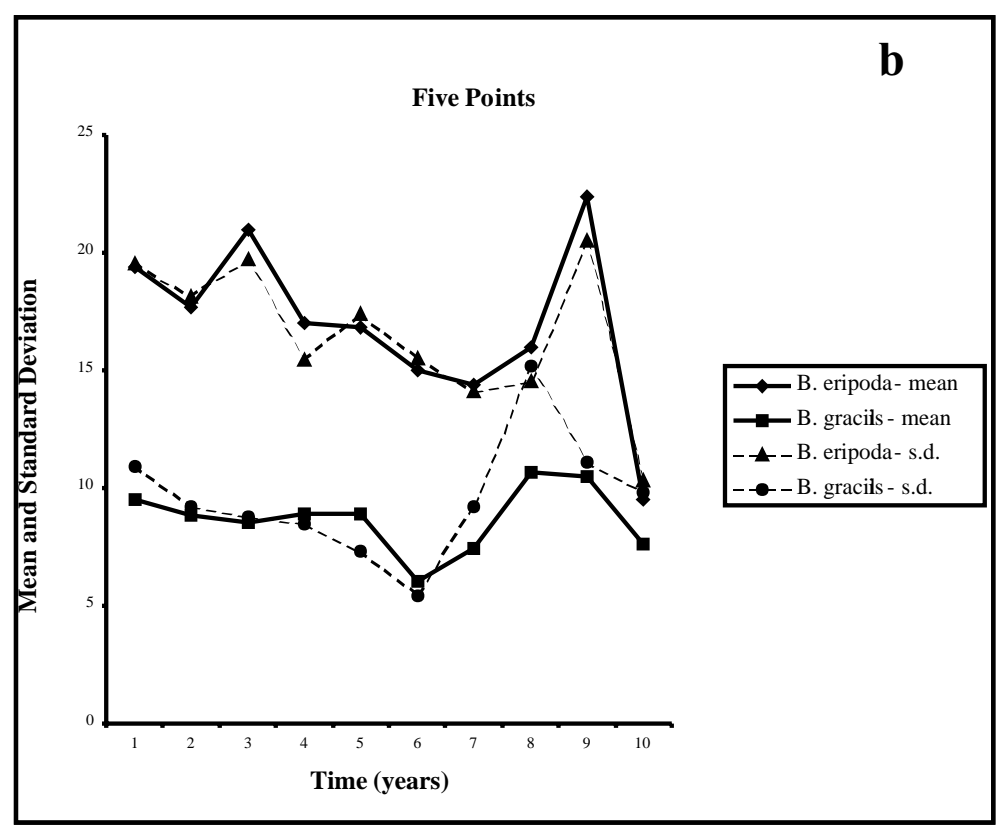

positive autocorrelation. When $H$ is less than 0.5 , the structure shows anti-persistence, or long-range negative autocorrelation. The Hurst exponent is related to the fractal dimension $D$ through $D=2-H$ (Mandelbrot 1983).

The Hurst exponent was fairly consistent in time for both species. The exponent for $B$. eripoda ranged from 0.345 to 0.41 in Deep Well and from 0.33 to 0.39 in Five Points. The exponent for $B$. gracilis ranged from 0.35 to 0.375 in Deep Well and $0.345-0.405$ in Five Points. These results indicate that the fractal structures of fluctuations for both species are anti-persistent. It is interesting to note that species showed higher temporal variation in the Hurst exponent in sites further from their normal dis- tributional limits. The autocorrelation dynamics of both species in both sites are shown in Figure 7 for a typical year. In both sites, the two species show fairly similar autocorrelation dynamics at low resolution (Block size 116), but then as resolution becomes higher, dynamics begins to diverge. As expected by the low Hurst exponent, both species showed significant negative correlations at high lag.

\section{Discussion}

We set out to examine spatial pattern in dominant species at different points along a biome transition zone. Direct observation of spatial pattern in terms of patch size 

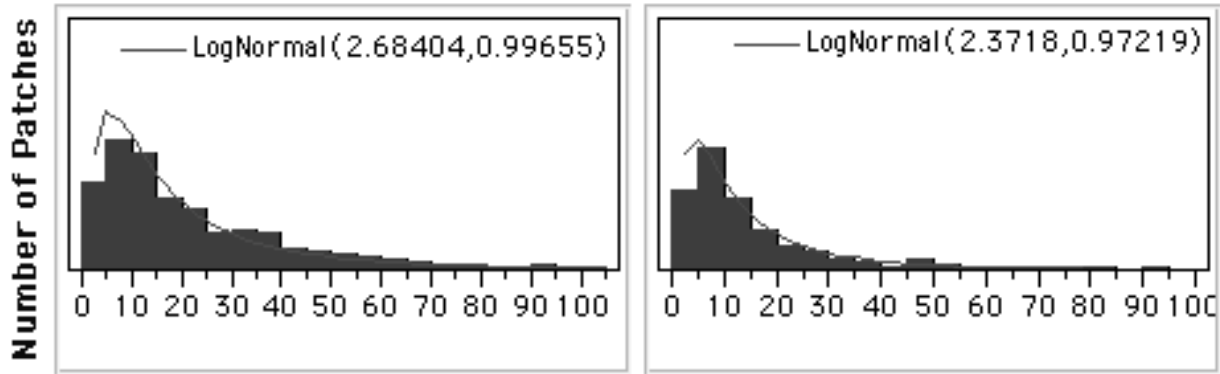

\section{Size of Patch}

Figure 5. Inter-patch (distance between patches) distribution in 1989. Inter-patches are those in which either both or the other dominant grass is absent. Abscissa is inter-patch size classes which are the same patch size classes (see Figure 3).

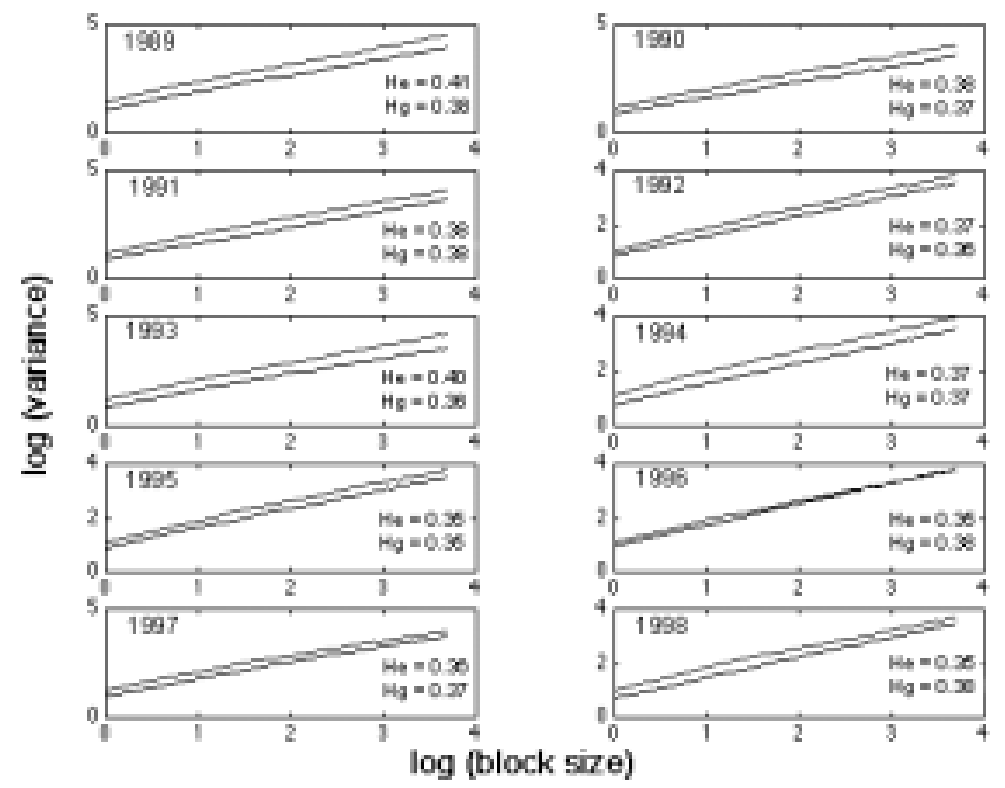

a

Figure 6. The relationship between variance and block size in Deep Well (a) and Five Points (b). Block size ranges from $1(10 \mathrm{~cm})$ to $40(400$ $\mathrm{cm})$. The top lines on the graph are $B$. eripoda and the bottom lines, $B$. gracilis.

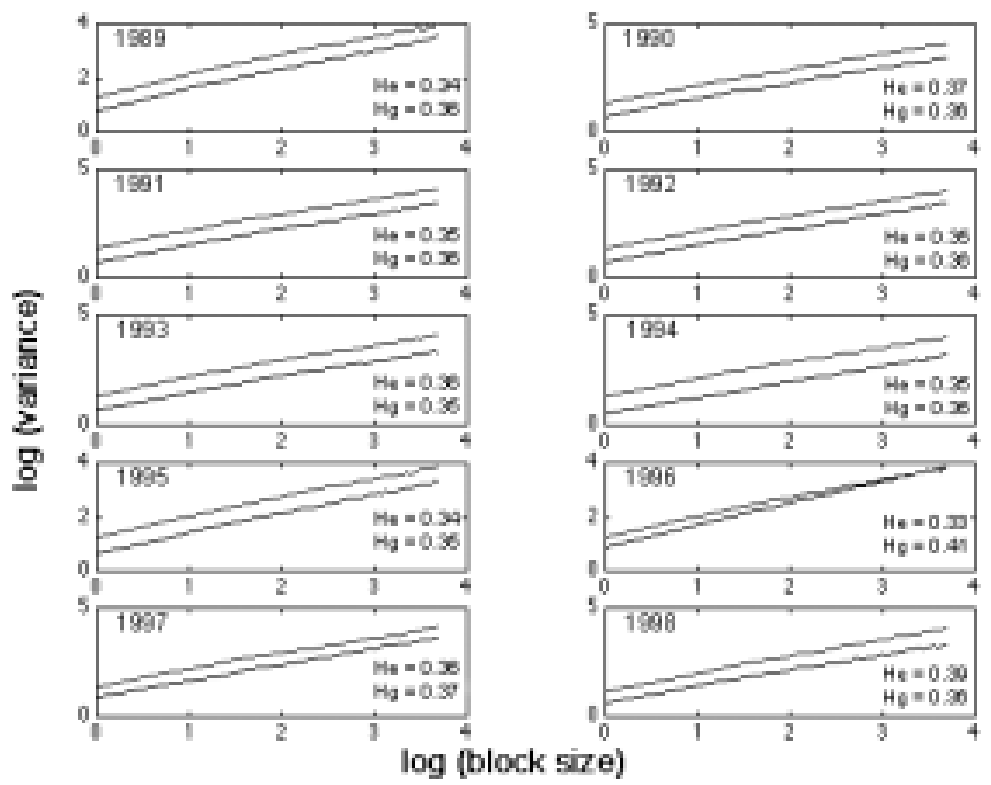


$\mathbf{a}$

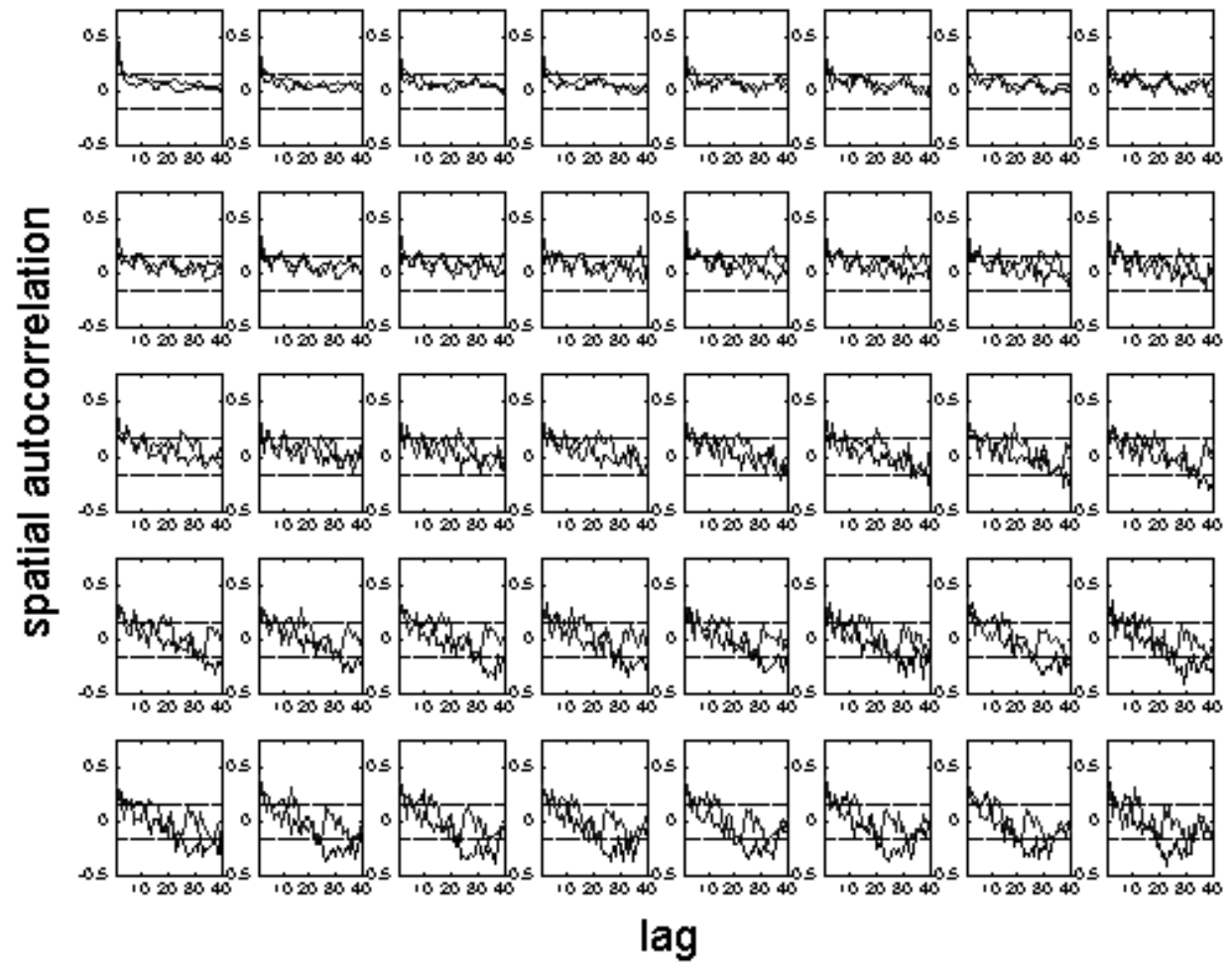

b

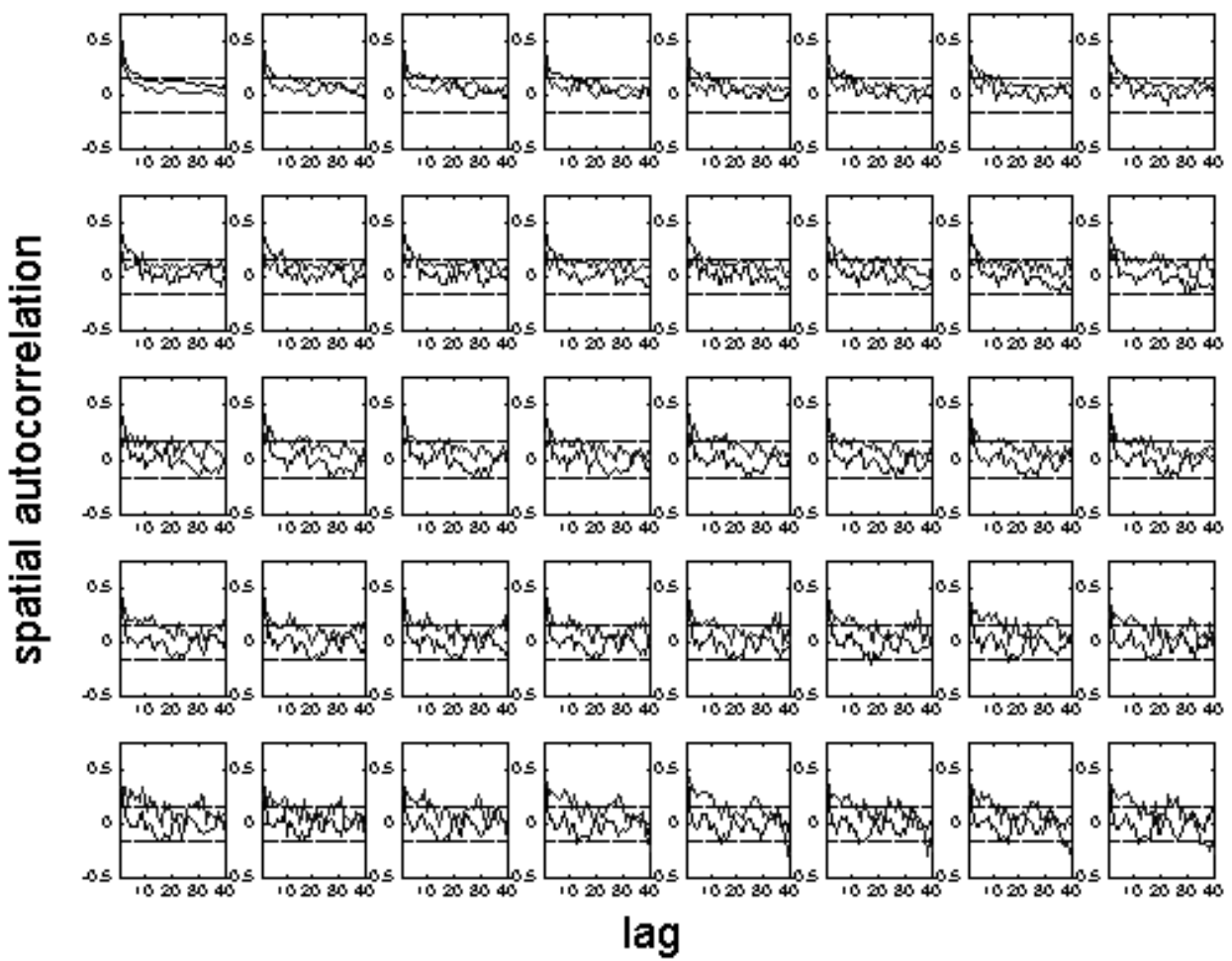

Figure 7. Spatial autocorrelation dynamics of B. eripoda (predominantly lower/bottom graph) and B. gracilis (predominantly higher/top graph) at different block sizes (Block size 1-top left; Block size 40-bottom right). Straight lines are conservative confidence intervals for positive and negative autocorrelation. The year is 1989. Deep Well is in (a) and Five Points is in (b). Abscissa of each graph is lag (distance between compared points ranging from $1(10 \mathrm{~cm})$ to $40(400 \mathrm{~cm})$. 
distribution revealed that response to movement away from their distributional limit was time-dependent and species-specific. The characteristic patch size could be interpreted as the most commonly occurring patch size. In all cases, this was very small (around 10-30 cm in width). Patch size of populations is, however, only one part of the spatial pattern. The other is of course how those patches are distributed in space. For this, examination of the distribution of patches in space (inter-patch distance) was important and revealing. These were similar for both species, but in general, inter-patch distance was even smaller than patch size. Why is this important? Because these two components of spatial pattern can be confounded when using pattern detection techniques such as correlograms. We were thus interested to see the results of the analytical techniques.

Classical variance vs. block size analysis gave a result different from those of patch size distribution analysis: 1 . no characteristic spatial resolution (quadrat size), but rather a fractal structure of spatial variation in abundance and 2. a tendency towards consistency of the pattern in time when species were closer to their distributional limit. All Hurst exponents were found to be less than 0.5, indicating anti-persistence. These results are in general agreement with the results obtained by spectral analysis and detrended fluctuation analysis (Wang 2001). This implies that spatial patterns of both species are aggregated; aggregation may be regarded as special type of resource partitioning which promotes coexistence (Ives 1991). Autocorrelation analysis performed at different spatial resolution produced results consistent with the fractal interpretation of variance analysis, but not clearly interpretable on their own. We found that autocorrelation dynamics was generally affected by quadrat size (in agreement with Qi and Wu 1996 and Fortin 2000). We feel that this may be due to the fact that this method is more sensitive to small inter-patch distance than block variance techniques, but this remains to be formalized and tested.

Regarding the ecology of spatial dynamics of species along an ecotone, we can compare our results with those of Gosz (1993) and Aguilera and Lauenroth (1993). The latter found that the Central Plains Experimental Range (CPER), in which B. gracilis accounts for over $90 \%$ of plant cover, patch sizes were much higher than the ones found in the ecotonal sites. Gosz (1993) found decreasing patch size as the distributional limits of species are reached and hypothesized that this was due to increased sensitivity to microsite conditions at the range limits of a species. We found this to be the case as well, but interpret it more in terms of structural dynamics as reflected in the higher variation in the Hurst exponent in time and stand- ard deviation of patch size. We hypothesize that this is due to the fact that in transition zones, species interactions may not yet have had time to stabilize. When species were fairly close to their distributional limits, spatial pattern tended to be highly organized (Hurst exponent consistent in time, lower standard deviation in patch size), and although we do not have physical environmental data to make the case, we feel that this organization is likely linked to the way these species colonize space through clonal growth. We know in general that the study sites display considerable fine-scale heterogeneity in terms of soil characteristics and animal disturbance. We think that even if it could be determined that these were causes for species patterns, this would not diminish the results presented here.

In conclusion, we found that raw observation of spatiotemporal abundance and patch size distribution provided too fine a resolution to detect spatial organization as an emergent property in ecotonal dynamics. The latter was detectable by scaling of spatial variation through quadrat pooling but was not obvious from autocorrelation analyses. We feel that the two components of spatial variation (patch size and inter-patch size) could have been confounded by autocorrelation analyses. The results inspire, at least in us, future work to examine the theoretical relationships between these components of spatial variation, their contribution to power law scaling behaviour and their implications in ecology.

Acknowledgments: X.F. Wang provided computational support. Funding support was from an NSERC of Canada grant to M.A. and U.S. National Science Foundation (DEB-00-80529, DEB-00-83422 and DEI-98-20318) and DOE/Sandia National Laboratories (BG-7557) grants to B.L. We gratefully acknowledge critical comments from S. Bartha and J.B. Wilson. This is Sevilleta publication no. 220.

\section{References}

Aguilera, M.O. and W.K. Lauenroth. 1993. Neighborhood interactions in a natural population of the perennial bunchgrass Bouteloua gracilis. Oecologia 94:595-602.

Berry, M. V. and Z.V. Lewis. 1980. On the Weirstrass-Mandelbrot fractal function. Proc. Royal Soc. London Series A 370:459-484.

Burrough, P.A. 1981. The fractal dimension of landscape and other data. Nature 294:240-242.

Castri di F., A.J. Hansen and M.M. Holland. 1988. A new look at ecotones: emerging international projects on landscape boundaries. Biology International 17: 47-106.

De Patta Pillar, V. 2001. MULTIV. Multivariate Exploratory Analysis, Randomization Testing and Bootstrap Resampling. Universidade Federal Rio Grande do Sul, Porto Alegre, Brazil.

Delcourt, H.R. and P.A. Delcourt and W. Thompson. 1983. Dynamic plant ecology: the spectrum of vegetational change in space and time. Quaternary Science Reviews 1:153-175.

Fortin, M.J. 1994. Edge detection algorithms for 2-dimensional ecological data. Ecology 75:956-965. 
Fortin, M.J. 2000. Effects of sampling unit resolution on the estimation of spatial autocorrelation. Ecoscience 6:636-641.

Gosz, J.R. 1991. Sevilleta. In: K. Van Cleve and S. Martin (eds.), Long-Term Ecological Research in the United States. LongTerm Ecological Research Network Office, University of Washington, Seattle, WA. pp.148-157.

Gosz, J. R. 1993. Ecotone hierarchies. Ecological Applications 3:369-376.

Gosz, J.R. and P.J.H. Sharpe. 1989. Broad-scale concepts for interactions of climate, topography, and biota at biome transitions. Landscape Ecology 3:229-243.

Greig-Smith, P. 1979. Pattern in vegetation. J. Ecol. 67:755-779.

Greig-Smith, P. 1983. Quantitative Plant Ecology. 3rd ed. Blackwell Scientific, Oxford

Hurst, H.E. 1951. Long term storage capacity of resevoirs. Transactions of the American Society of Civil Engineers 116:770-799.

Ives, A.R. 1991. Aggregation and coexistence in a carrion fly community. Ecol. Monogr. 61: 75-94.

Li, B.L. 2000. Fractal geometry applications in description and analysis of patch patterns and patch dynamics. Ecol. Model. 132: 33-50.

Li, B.L. 2001a. Applications of fractal geometry and percolation theory to landscape analysis and assessments. In: M.E. Jensen and
P.E. Bourgeron (eds.), A Guidebook for Integrated Ecological Assessments. Springer, New York. pp. 200-210.

Li, B.L. 2001b. A theoretical framework of ecological phase transitions for characterizing tree-grass dynamics. Acta Biotheoretica, in press.

Loehle, C., B.L. Li and R.C. Sundell. 1996. Forest spread and phase transitions at forest-prairie ecotones in Kansas, USA. Landscape Ecology 11: 225-235.

Mandelbrot, B.B. 1983. The Fractal Geometry of Nature. W.H. Freeman, San Francisco.

Peitgen, H.O., H. Jürgens and D. Saupe. 1992. Chaos and Fractals: New Frontiers of Science. Springer, New York.

Qi, Y. and J. Wu 1996. Effects of changing spatial resolution on the results of landscape pattern analysis using spatial autocorrelation indices. Landscape Ecol. 11:39-49.

Upton, G.J.G. and B.Fingleton. 1985. Spatial Data Analysis by Example. Volume I. Point Pattern and Quantitative Data. Wiley and Sons, Chichester.

Wang, X.F. 2001. Temporal and spatial structures of black and blue gramas: statistical analysis of biotic and abiotic interactions from Sevilleta vegetation transect data. M.Sc. Thesis. University of New Mexico, Albuquerque, New Mexico, USA. 